\title{
LES modeling of piloted jet flames with inhomogeneous inlets using tabulated chemistry methods
}

Conference Paper · January 2017

Dol: $10.2514 / 6.2017-1471$

CITATION

1

5 authors, including:

Giampaolo Maio

CentraleSupélec

3 PUBLICATIONS 4 CITATIONS

SEE PROFILE

Renaud Mercier

SAFRAN Tech

24 PUBLICATIONS 95 CITATIONS

SEE PROFILE

Some of the authors of this publication are also working on these related projects:

Project Plasma-assisted combustion View project

Project PERCEVAL - Powering The Future with Clean and Future Aero-Engines View project
READS

65

BENOIT FIORINA

CentraleSupélec

74 PUBLICATIONS 1,193 CITATIONS

SEE PROFILE

Vincent Moureau

CORIA

109 PUBLICATIONS 1,498 CITATIONS

SEE PROFILE 


\title{
LES modeling of piloted jet flames with inhomogeneous inlets using tabulated chemistry methods
}

\author{
Giampaolo Maio ${ }^{1}$ Mélody Cailler ${ }^{1,2}$ Renaud Mercier ${ }^{2}$ Vincent Moureau $^{3}$ Benoît Fiorina ${ }^{1}$ \\ ${ }^{1}$ Laboratoire EM2C, CNRS, CentraleSupélec, Université Paris-Saclay, \\ Grande Voie des Vignes, 92295 Châtenay-Malabry cedex, France \\ ${ }^{2}$ SAFRAN Tech, Rue des Jeunes Bois, 78772 Magny les Hameaux, FRANCE \\ ${ }^{3}$ Normandie Univ, INSA Rouen, UNIROUEN, CNRS, CORIA, 76000 Rouen, France
}

\begin{abstract}
The present work focuses on the LES of piloted jet flame with inhomogeneous inlets experimented at both Sydney University and Sandia National Laboratories. This flame configuration is very challenging because various flame regimes are encountered by changing the burner configuration. Two turbulent combustion models that both rely on chemistry tabulation for premixed flamelets are challenged: F-TACLES model (Filtered TAbulated Chemistry for LES) and TFLES-FPI model (Thickened Flame model for LES coupled with FPI tabulation). A comparison between the numerical results and the experimental data is performed for two inlets of reactants configurations: at the burner exit plane, the first one presents an homogeneous mixing, whereas the second one exhibits a strong gradient of equivalence ratio. A good agreement is observed between experiments and the two simulations when focusing on conditional mean of temperature. However, differences are evidenced when analyzing the radial profiles. The main reason of the discrepancies are certainly due to the difficulty to capture accurately the mixing between the main jet and the co-flow stream. This effect is more pronounced for the case with inhomogeneous inlets.
\end{abstract}

\section{Introduction}

LES is nowadays a key tool to predict the efficiency of aeronautical combustion chambers. However, despite the tremendous progress in supercomputing, grids used in practical industrial configurations remain typically coarser than the flame thickness and small scale flame wrinkling patterns. Consequently chemical species cannot be resolved and subgrid scale modeling is needed for the flame structure and its interactions with the turbulence. As discussed in the recent review of combustion chemistry modeling for LES, ${ }^{1}$ different methods have been developed to reduce the coputational cost of complex chemistry. Among them tabulated chemistry is included. Tabulated chemistry is indeed a reduced order method to include complex chemistry phenomena in CFD simulations. This strategy makes an a priori assumption about the flame structure allowing, in the preprocessing phase, the pretabulation of all thermochemical quantities as a function of a redused set of variables.

In addition, in LES of premixed and stratified combustion (here "stratification" denotes local equivalence ratio heterogeneities) dedicated methods are required to capture the propagation of the filtered wrinkled flame fronts, underresolved in practical grids. A first approach is the Thickened Flame Model (TFLES), which artificially thickens the flame front by modifying the diffusion coefficient and the pre-exponential constant. ${ }^{2}$ This approach is robust and has been applied to complex combustors, using tabulated chemistry techniques for adiabatic ${ }^{3}$ and non-adiabatic flames. ${ }^{4}$ An alternative to TFLES is to explicitly filter chemical reaction rate at a size greater than the grid spacing so that the filtered flame front is well resolved. This strategy has been coupled with tabulated chemistry through the Filtered Tabulated Chemistry for LES (F-TACLES) model. ${ }^{5}$ The F-TACLES formalism has been developed to capture the filtered flame propagation in complex reactive flow configurations, such as stratified ${ }^{6}$ or non-adiabatic flames. ${ }^{7}$

F-TACLES and TFLES models have been already widely challenged for premixed and weakly stratified premixed flames. $^{8,9}$ However, in many practical applications, the fuel/air mixture issuing the injection system exhibits strong composition inhomogeneities, promoting the development of multiple flame structures. ${ }^{10}$ 
For example, in gas turbine engines, the fuel stream is not perfectly mixed with air stream at the inlet of the chamber and in addition recirculation of burnt gases could impact on the fresh mixture. ${ }^{11}$ All these phenomena lead to locally strong inhomogeneities in equivalence ratio promoting the development of mixed combustion regimes. ${ }^{12}$ It has been recently observed that combustion models have to account for the coexistence of both premixed and non-premixed regimes to predict pollutant such as CO. ${ }^{13}$ However, existing turbulent combustion models based on chemistry reduction methods are in general optimized to a specific combustion regime ${ }^{1}$ and fail to capture complex flame structure.

Sydney modified piloted burner, ${ }^{14}$ recently studied experimentally both in Sandia and in Sydney laboratories, is a challenging configuration because it allows to stabilize multiple combustion regimes (stratified, premixed and diffusion mode) changing the experimental set-up. LES of such flame configuration have been already conducted using a revised FPV model, ${ }^{15}$ a multi-regime flamelet model ${ }^{16}$ and MMC-LES model. ${ }^{17}$ Such models account for pretabulation of non-premixed flamelets or are optimized for non-premixed flames modeling. The aim of the present paper is instead to estimate whether approaches based on premixed flamelets (TFLES and F-TACLES) can be extended to multi-mode combustion regimes. To estimate such capability LES of Sydney modified piloted burner is conducted.

\section{Combustion modeling}

\section{A. Chemistry tabulation}

In the present work combustion chemistry modeling relies on chemistry tabulation using premixed flamelet archetypes. For such purpose a set of 1-D freely propagating premixed flames is computed using the detailed chemistry GRI.3.0 ${ }^{18}$ mechanism, involving 53 species and 325 reactions and considering differential diffusion effects. The 1-D flames are computed using the REGATH ${ }^{19}$ thermochemistry package developed at the EM2C laboratory. The fresh gas equivalence ratio is varied between the full flammability limits ( i.e $0.5<$ $\phi<1.8$ ). Following the FPI formalism introduced by Gicquel et al. in, ${ }^{20}$ the chemical subspace covered by the 1-D flames is mapped as a function of two coordinates: the mixture fraction $Z$ and the progress variable $Y_{c}$. The mixture fraction is used to identify the fresh gas equivalence ratio, varying from 0 in pure air and 1 in pure fuel. While the progress variable $Y_{c}$, defined as a linear combination of species mass fractions, describes the progress of the reaction. In the present work $Y_{c}$ is $Y_{C O}+Y_{\mathrm{CO}_{2}}$ as supported by Fiorina et al. $^{21}$ All thermochemical quantities $\psi$ are tabulated as a function of $Y_{c}$ and $Z$, so that $\psi\left[Y_{c}, Z\right]=\psi^{*}$, where for all the terms the superscript $*$ denotes quantities computed from 1-D premixed flamelets. Two balance equations are solved to couple tabulated chemistry with the LES flame solver: one for the filtered mixture fraction $\widetilde{Z}$ and the other one for the filtered progress variable $\widetilde{Y}_{c}$. The following filtered scalar transport equation is considered for the mixture fraction variable:

$$
\frac{\partial \bar{\rho} \widetilde{Z}}{\partial t}+\frac{\partial}{\partial x_{i}}\left(\bar{\rho} \widetilde{u_{i}} \widetilde{Z}\right)=\frac{\partial}{\partial x_{i}}\left(\left[\frac{\bar{\mu}}{S c}+\frac{\mu_{t}}{S c_{t}}\right] \frac{\partial \widetilde{Z}}{\partial x_{i}}\right)
$$

Where $\rho$ and $u_{i}$ are the flow density and velocity in i direction, $\mu$ and $\mu_{t}$ are respectively the laminar and the turbulent viscosity; $S c$ and $S c_{t}$ are respectively the laminar and the turbulent Schmidt numbers. $Z$ is treated as a non-reactive scalar ${ }^{22}$ and the unresolved sub-grid convective fluxes are closed using a gradient assumption, as detailed in. ${ }^{23}$ Instead for the progress variable $Y_{c}$ the formalism of the filtered balance equation and the closure of the unresolved terms differ between TFLES and F-TACLES strategies as detailed below.

\section{B. TFLES-FPI model}

TFLES handles the under-resolution of the flame front in the LES grid using an artificially thickening of the flame front. In the framework of premixed flamelets based tabulation (FPI) a thickening factor $F_{Y_{c}}$ is applied to both diffusion and the source term of the filtered Progress variable balance equation as detailed in Eq. 2.

$$
\frac{\partial \bar{\rho} \widetilde{Y}_{c}}{\partial t}+\frac{\partial}{\partial x_{i}}\left(\bar{\rho}{\widetilde{u_{i}}}_{\tilde{Y}_{c}}\right)=\frac{\partial}{\partial x_{i}}\left(\left[\alpha_{Y_{c}} F_{Y_{c}} \Xi_{\Delta} \frac{\bar{\mu}}{S c}+(1-\Gamma) \frac{\mu_{t}}{S c_{t}}\right] \frac{\partial \widetilde{Y}_{c}}{\partial x_{i}}\right)+\frac{\Xi_{\Delta} \widetilde{\tilde{\dot{\omega}}_{Y_{c}}}}{F_{Y_{c}}}
$$


Where $\Xi_{\Delta}$ is the subgrid wrinkling flame surface, $F_{Y_{c}}$ the thickening factor, $\Gamma$ the flame sensor and $\widetilde{\dot{\omega}_{Y_{c}}}$ the filtered progress variable source term. The progress variable source term is tabulated as a function of $Z$ and $Y_{c}$ (i.e. $\dot{\omega}_{Y_{c}}\left[Y_{c}, Z\right]=\dot{\omega}_{Y_{c}}^{*}$ ). As well as the dynamic viscosity $\mu$ (i.e. $\mu\left[Y_{c}, Z\right]=\mu^{*}$ ). The parameter $\alpha_{Y c}$ which takes into account the differential diffusion effects in the direction normal to the flame front is defined as follows:

$$
\alpha_{Y_{c}}[\widetilde{Y c}, \widetilde{z}]=-\frac{\sum_{k=1}^{N_{s} p} n_{k} \rho^{*} Y_{k}^{*} V_{k}^{*}}{\frac{\mu}{S c} \frac{\partial \widetilde{Y}_{c}^{*}}{\partial x_{i}^{*}}}
$$

The flame surface wrinkling $\Xi_{\Delta}$ is modeled using the Charlette et al. formulation ${ }^{24}$ including Wang et al. ${ }^{25}$ corrections. The flame sensor $\Gamma$ is applied to detect the reaction zone position:

$$
\left\{\begin{array}{c}
\Gamma=1 \quad \text { if } \quad \omega_{Y_{c}}>\omega_{Y_{c}}^{0} \\
\Gamma=0
\end{array}\right.
$$

where $\omega_{Y_{c}}^{0}$ is a threshold value defined as:

$$
\omega_{Y_{c}}^{0}=\frac{\operatorname{Max}\left(\omega_{Y_{c}}\left[Y_{c}, Z\right]\right)}{100}
$$

As discussed by Franzelli ${ }^{26}$ the flame sensor is then filtered to avoid numerical problems. A grid adaptive thickening factor is defined by introducing the flame sensor as follows:

$$
F_{Y_{c}}=1+\left(F_{\max }-1\right) \Gamma
$$

where $F_{\max }$ reads:

$$
F_{\max }=n \frac{\Delta_{x}}{\delta_{l_{0}}}
$$

where $\delta_{l_{0}}$ is the stoichiometric laminar flame thickness, $\Delta_{x}$ is the local cell size and $n$ is the minimum number of points required across the flame front to correctly resolve the flame structure.

\section{F-TACLES model}

The second turbulent combustion model, used for the LES simulation of the Sydney piloted jet burner, is F-TACLES (Filtered Tabulated Chemistry for LES) model. F-TACLES is based on the tabulation of a set of 1-D freely propagating premixed flames, filtered using a Gaussian operator of width $\Delta$ along the direction normal to the flame front. $\widetilde{Y}_{c}$ is solution of the following balance equation :

$$
\frac{\partial \bar{\rho} \widetilde{Y}_{c}}{\partial t}+\frac{\partial}{\partial x_{i}}\left(\bar{\rho} \widetilde{u_{i}} \widetilde{Y}_{c}\right)=\frac{\partial}{\partial x_{i}}\left(\alpha_{Y c} \rho_{0} D_{0} \frac{\partial \widetilde{Y}_{c}}{\partial x_{i}}\right)-\frac{\partial}{\partial x_{i}}\left(\bar{\rho} \widetilde{u_{i} Y_{c}}-\bar{\rho} \widetilde{u_{i}} \widetilde{Y}_{c}\right)+\Xi_{\Delta} \widetilde{\rho} \widetilde{\dot{\omega}_{Y_{c}}}
$$

The parameters $D_{0}$ and $\rho_{0}$ are reference parameters. The factor $\alpha_{Y_{c}}$ is expressed following the same formalism proposed in Eq 3, modeling the molecular diffusive-fluxes using data from the filtered 1-D flamelets as follows:

$$
\alpha_{Y_{c}}[\widetilde{Y c}, \widetilde{z}]=-\frac{\overline{\sum_{k=1}^{N_{s p}} n_{k} \rho^{*} Y_{k}^{*} V_{k}^{*}}}{\rho_{0} D_{0} \frac{\partial \widetilde{Y}_{c}^{*}}{\partial x_{i}^{*}}}
$$

According to ${ }^{5}$ the unresolved sub-grid fluxes are modeled as follows :

$$
\frac{\partial}{\partial x_{i}}\left(\bar{\rho} \widetilde{u_{i} Y_{c}}-\bar{\rho}{\widetilde{u_{i}}}_{Y_{c}}\right)=\Xi_{\Delta} \Omega_{Y_{c}}\left[\widetilde{Y}_{c}, \widetilde{z}\right]+\frac{\partial}{\partial x_{i}}\left(\left(\Xi_{\Delta}-1\right) \alpha_{Y c} \overline{\rho D} \frac{\partial \widetilde{Y}_{c}}{\partial x_{i}}\right)
$$

where $\Omega_{Y_{c}}$, is computed and tabulated from the filtered database as:

$$
\left.\Omega_{Y_{c}}\left[\widetilde{Y}_{c}, \widetilde{z}\right]=\overline{\rho^{*}(z) S_{l}^{*}(z) \frac{\partial Y_{c}^{*}}{\partial x_{i}^{*}}}-\rho_{(} z\right)^{*} S_{l}^{*}(z) \frac{\partial \widetilde{Y_{c}^{*}}}{\partial x_{i}^{*}}
$$

The filtered reaction rate is estimated from the filtered look-up table as:

$$
\tilde{\dot{\omega}}_{Y_{c}}\left[\tilde{Y}_{c}, \widetilde{z}\right]=\widetilde{\dot{\omega}}_{Y_{c}}^{*}
$$

Further details about the F-TACLES model and about the generation of the filtered database can be found in $^{5}$ and in. ${ }^{27}$ 


\section{Experimental set-up of the turbulent jet flame}

The burner assembly shown in Fig. 1 consists of two concentric tubes surrounded by a pilot annulus. It is centered in a wind tunnel supplying a co-flowing air stream at fixed velocity. A set of experimental data has been provided by Sydney university ${ }^{28}$ and Sandia Laboratory. ${ }^{14}$ In the configuration analyzed in this paper, the central tube is fed with fuel whereas air is flowing through the outer tube. The air co-flow velocity is fixed at $15 \mathrm{~m} / \mathrm{s}$. The central pipe can be recessed upstream of the burner exit plane changing $L_{r}$, varying therefore the mixing between fuel and air. For a sufficiently large recession distance the mixture is nearly homogeneous at the burner exit, while intermediate recession distances lead to equivalence ratio inhomogeneities.

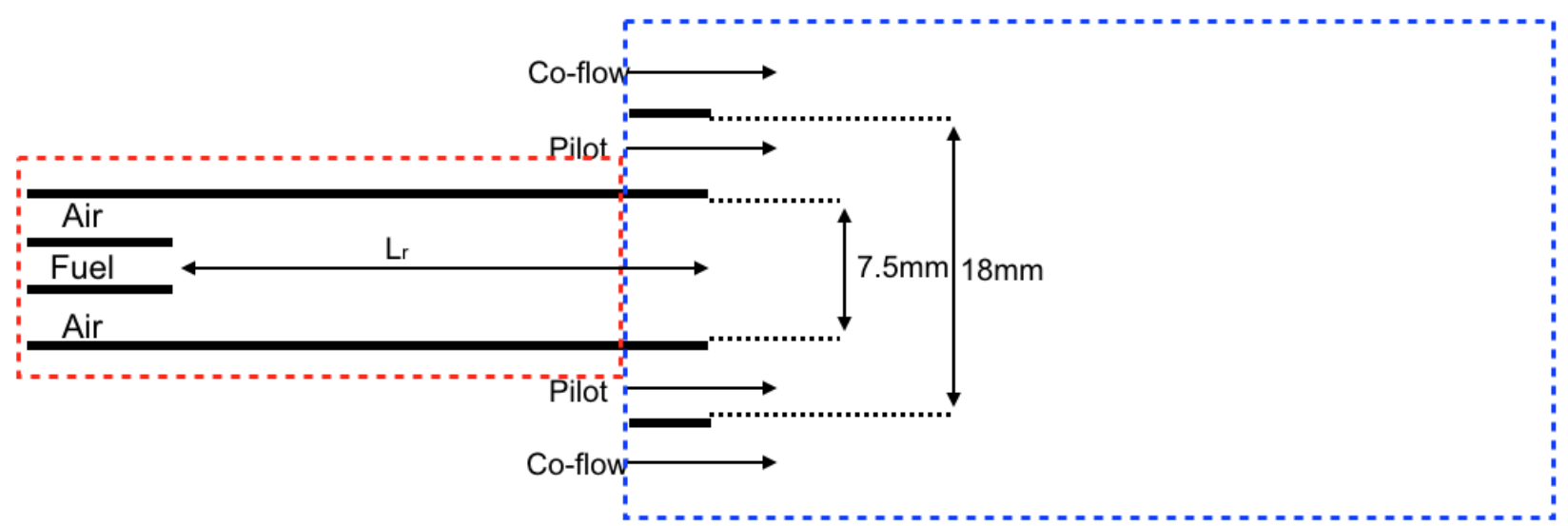

Figure 1. Schematic 2D cutway of the burner. In red is underlined the non-reacting fluel/air mixing computational domain simulated by Princeton university. ${ }^{15}$ While in blue is underlined the reacting computational domain simulated in the present work.

Two configurations have been selected for the numerical simulations: the first one features an homogeneous composition in equivalence ratio at the burner exit plane $(L r=300 \mathrm{~mm})$, while the second exhibits inhomogeneous mixture fraction profile $(L r=75 \mathrm{~mm})$. Both configurations are at $70 \%$ from the blow-off velocity limit. Using the notation coming from the experimental study the two configuration simulated are named as FJ200-5GP-Lr300-59 and FJ20-5GP-Lr75-80. FJ200 refers to the condition where the fuel is fed from the inner tube and the air is fed through the outer tube, with a volumetric air to fuel ratio $V_{A} / V_{F}$ equal to 2 . The label 5GP corresponds to the five-gas pilot mixture, which is a stoichiometric mixture of acetylene, hydrogen, oxygen, nitrogen and carbon dioxide. The pilot mixture has been designed to have the same $\mathrm{C} / \mathrm{H}$ ratio and adiabatic flame temperature as the methane/air mixture at stoichiometry. As shown in Fig. 1, $L_{r}$ refers to the recession distance of the inner tube in respect to the burner exit plane. 59 and 80 are respectively the bulk jet velocities of the two test-cases expressed in $\mathrm{m} / \mathrm{s}$. As detailed in the experimental study proposed by Meares et al. and Barlow et al., the configuration exhibiting inhomogeneity in equivalence ratio at the burner exit plane is more stable than the homogeneous one. In this paper, it will be often used the shortened form Lr300-59 or homogeneous case to make reference to the configuration FJ200-5GP-Lr300-59, and the the shortened form Lr75-80 or inhomogeneous case to make reference to the configuration FJ20-5GP-Lr75-80.

\section{Numerical set-up}

The calculations are performed with the unstructured finite-volume low Mach number code YALES2 designed by Moureau etal. ${ }^{29}$ A centered fourth-order scheme in space and time is used for the numerical integration of the convective terms. The sub-grid Reynolds stresses tensor is closed with the Dynamic Smagorinsky model. ${ }^{30}$ The reactive computational domain shown in Fig 1 and Fig 2, in the axial direction starts from 1D diameter upstream the burner exit plane (D is the main jet diameter) up to 40D downstream the burner exit plane. In the radial direction, the computational domain extends to 20D. The mesh consists of 72 millions of tetrahedral elements. In the flame zone the mean cell size evolves from $0.1 \mathrm{~mm}$ to $0.5 \mathrm{~mm}$ at the end of the domain.

Non-reactive LES of the mixing process between fuel and air in the mixing tube has been performed by 


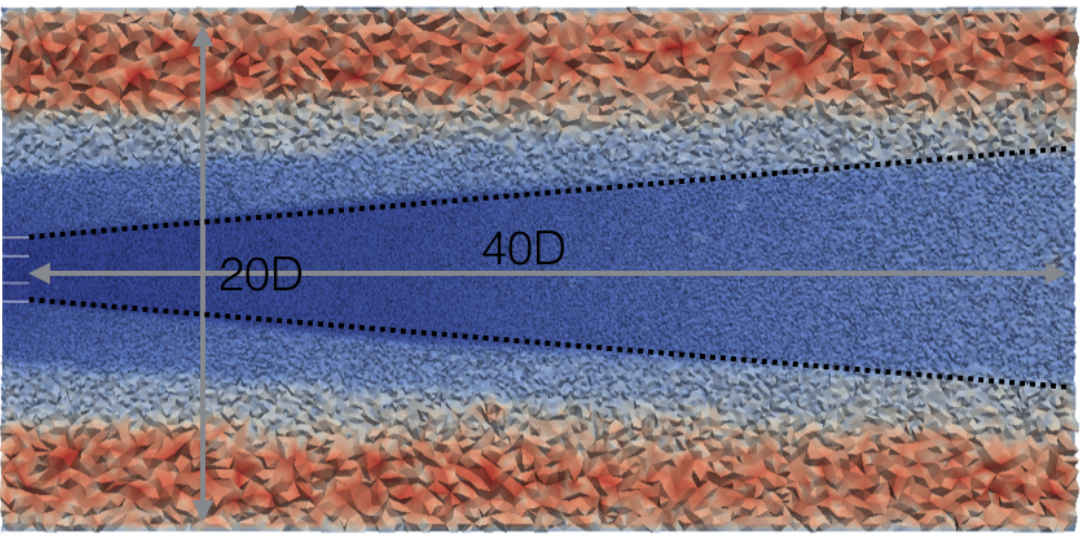

Figure 2. 2-D view of the mesh colored by the cell size dimension in the centerline plane. The computational domain extends from 1-D upstream the burner exit plane to $40 \mathrm{D}$ downstream the burner exit plane. The black dashed lines delimit the refined mesh zone that corresponds to the flame zone.

Princeton University (the computational domain is defined in Fig.1). Two configurations have been investigated that correspond to $L r=300 \mathrm{~mm}$ and $L r=75 \mathrm{~mm}$ distances, respectively. Unsteady solutions of these simulations constitute a numerical database showed to the numerical combustion community within the framework of the TNF workshop. ${ }^{31}$ This database is used to prescribe the main inlet boundary conditions, built in term of flow velocity and mixture fraction.

Computed mean mixture fraction and velocity profiles at the burner exit plane are shown in Fig $3 \mathrm{a}$ and $\mathrm{b}$. The case $L r 300$ - 59 exhibits quasi-uniform mixture fraction profile, that corresponds to a mean equivalence ratio of 4.76 , which is above the rich flammability limit $(\phi=1.8)$. A diffusion flame structure is then expected. At the opposite, the $L r 75-80$ case exhibits a strongly "inhomogeneous" mixture fraction profile at the burner exit plane. The fresh equivalence ratio within the main-jet reaches locally flammable conditions in the shear layer between the main jet and the pilot stream. So stratified flame structures are therefore expected in the flame anchoring zone, while further downstream the non-premixed combustion mode will dominate the mean burner region, as discussed and detailed in. ${ }^{14}$ Figure $3 \mathrm{c}$ and $\mathrm{d}$ display computed rms values of velocity and mixture fration $1 \mathrm{D}$ downstream from the burner exit plane. As the mean bulk velocity is higher for the case $\operatorname{Lr} 75-80$, higher turbulent fluctuations are observed at the interface between the main jet and the pilot gases. The pilot gases are assumed to be composed of a stoichiometric $\mathrm{CH}_{4} / \mathrm{Air}$ mixture and the injection velocity is calculated to be consistent with the experimental fresh pilot mass flow rate.

The main characteristics of the two simulated configurations are summarized in Tab. 1

Table 1. Main characteristics of the two simulated flames including the bulk velocity of the three jets. $U_{b}$ is the mean bulk flow velocity of the main jet. $U_{p}$ and $T_{p}$ are respectively the pilot gases velocity and temperature. $U_{c}$ is the co-flow velocity.

\begin{tabular}{lcccccc}
\hline Flame & $L r[\mathrm{~mm}]$ & $U_{b}\left[{\left.\mathrm{~m} . \mathrm{s}^{-1}\right]}\right.$ & $U_{p}\left[{\left.\mathrm{~m} . \mathrm{s}^{-1}\right]}\right.$ & $T_{p}[\mathrm{~K}]$ & $U_{c}\left[{\left.\mathrm{~m} . \mathrm{s}^{-1}\right]}\right.$ & Main $\phi$ \\
\hline$L r-300-59$ & 300 & 59 & 25.62 & 2232 & 15.0 & 4.76 \\
\hline$L r-75-80$ & 75 & 80 & 25.62 & 2232 & 15.0 & 4.76 \\
\hline
\end{tabular}

For F-TACLES model, a filtered database has been generated with the filter size $\Delta=1 \mathrm{~mm}$. It corresponds to a stoichiometric filtered reactive layer $\widetilde{\delta_{r}} \approx 3.5 \delta_{l_{0}}$. As $\Delta_{x}$ has a mean value of $0.2 \mathrm{~mm}$ in the flame zone, the condition $n=\widetilde{\delta_{r}} / \Delta_{x}=5$ is satisfied, ensuring a proper propagation of the filtered flame front, without introducing numerical artifacts. As suggested in $^{32}$ for the TFLES-FPI simulations, a thickening factor of $F_{Y_{c}}=5 \Delta_{x} / \delta_{l_{0}}$ has been retained so that the thickened reactive layer $\overline{\delta_{r}}$ is equivalent to the filtered one retained for F-TACLES simulations. 


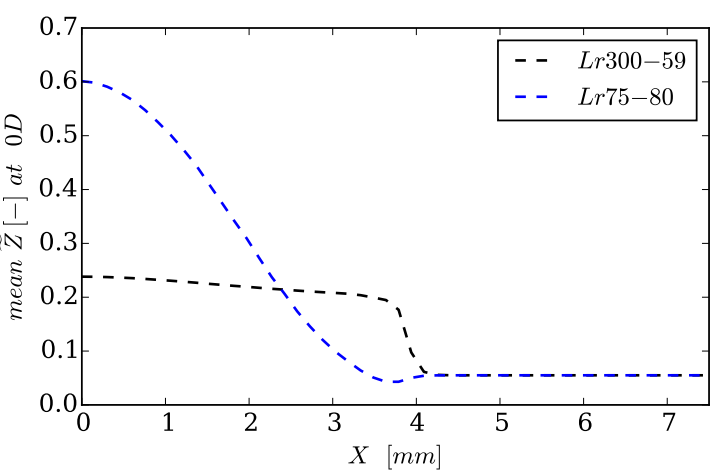

a)

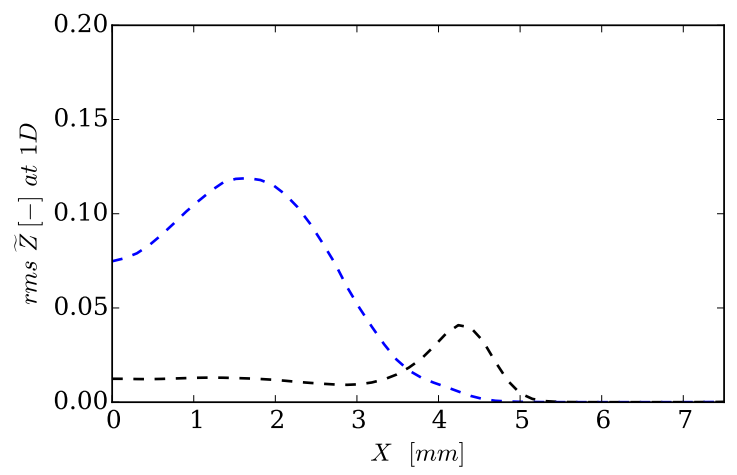

c)

Figure 3. a) Mean mixture fraction profile at burner exit plane for the two cases b) Mean axial velocity at the burner exit plane for the two cases. c) Root mean square values of mixture fraction fluctuations at 1D downstream the burner exit plane.c) Root mean square values of axial velocity fluctuations at 1D downstream the burner exit plane

\section{Simulation results}

The simulation results are compared against the experimental data. For the two simulated configurations, measurements of composition and temperature are available at several axial distances from the burner exit plane: $\mathrm{z} / \mathrm{D}=1, \mathrm{z} / \mathrm{D}=5, \mathrm{z} / \mathrm{D}=10, \mathrm{z} / \mathrm{D}=15, \mathrm{z} / \mathrm{D}=20$ and $\mathrm{z} / \mathrm{D}=30$.

Fig 4 shows the radial mean and RMS profiles of the mixture fraction and temperature for the homogeneous case (FJ200-5GP-Lr300-59). A comparison is performed between experimental data and the simulation results obtained using the two different combustion models TFLES-FPI and F-TACLES. The numerical results are close to the experiments for both mean and RMS quantities at the first two experimental radial positions $(\mathrm{z} / \mathrm{D}=1$ and $\mathrm{z} / \mathrm{D}=5)$. This indicates that the boundary conditions are well prescribed at the burner inlet. An overestimation of the "plateau" temperature between the pilot and the main jet is however observed. An analysis of the experimental data reveals that the discrepancies are due to the fact that the pilot gases do not fully reach equilibrium conditions before entering in the chamber, unlike to the numerical boundary condition assumption. The double peak in temperature and mixture fraction RMS at $\mathrm{z} / \mathrm{D}=1$ is also well captured by the numerical simulations. At $\mathrm{z} / \mathrm{D}=10$, significant discrepancies are observed in the mixing layer between measured and computed mixture fraction fields. This misprediction causes a shifting of the predicted peak temperature. Downstream $\mathrm{z} / \mathrm{D}=15$, the discrepancy in the mixing prediction is less evident mainly for F-TACLES combustion model. Regarding temperature the shifting of the peak towards the co-flow is still present. The overestimation of the temperature level on the center-line starting at $z=15 \mathrm{D}$ is more pronounced for the TF-FPI simulation than the F-TACLES simulations.

In Fig 5, a comparison is also conducted for the case FJ200-5GP-Lr75-80. The transition between the stratified premixed combustion mode to the diffusion flame mode, has been observed experimentally between 1D and 5D downstream the burner exit plane. This phenomenon is well captured numerically especially by the F-TACLES model. Unlike to the previous FJ200-5GP-Lr300-59 case, starting from 10D, the computations exhibits a too rapid dissipation of the mixture fraction gradients. The mixing returns 
well captured at 30D downstream. Mixture fraction profile biases also the positioning and the shape of the temperature profile.

To analyze the flame structure, instantaneous scatter plots of temperature as a function of the mixture fraction is plotted in Fig 6 for the homogeneous case and in Fig 7 for the inhomogeneous configuration. To underline the importance of populated regions, the plots are colored by the local normalized density of points. The numerical scatter plots are qualitatively in good agreement with the experimental data. The main trajectories described by numerical simulations and measurements are comparable, but the experimental data cover a broader range. For the homogeneous case, as pointed out in the experimental study, the nonpremixed flame structure dominates in the whole domain. Instead for the inhomogeneous case various flame structures can be identified. Near the burner exit at $\mathrm{z} / \mathrm{D}=1$ a stratified premixed combustion mode can be observed looking to steep temperature in correspondence to the stoichiometric mixture fraction zone; while further downstrean at $\mathrm{z} / \mathrm{D}=5$, the flame features a diffusion like structure. For $\mathrm{z} / \mathrm{D}=15$ and $\mathrm{z} / \mathrm{D}=30$, in both cases, extinction events, visible in the experiments, are also captured by simulations. The experimental trajectories are better captured by the F-TACLES simulation than the TFLES-FPI one especially in the richer flame zone. For both cases at $\mathrm{z} / \mathrm{D}=1$ high density of the points can be observed in correspondence to the stoichiometric line at high temperature values, such phenomenon is mainly representative of the pilot gases conditions. At the same axial location, on the rich side, high density of points is also visible at low temperature, corresponding to cold mixing between fuel and air. Further downstream, increasing of point density on the rich reacting branch can be noticed, pointing out an enlargement of the flame toward the centerline of the jet, where richer conditions are present. The non physical double band of points observed for the homogeneous case in the experiments at $\mathrm{z} / \mathrm{D}=1$ and $\mathrm{z} / \mathrm{D}=5$, on the rich side, is due to a slight asymmetry of the central tube positioning as remarked in the experimental study of the flame.

A quantitative comparison of the flame structure in the temperature/mixture fraction space is presented. Figure 8 plots the conditional mean temperature against mixture fraction mean for four different axial positions. For the homogeneous configuration, the flame structure is in good agreement with experimental results. For all axial positions, the temperature peak value and its position are well captured by numerical simulations. As already explained, the temperature peak misprediction at $\mathrm{Z} / \mathrm{D}=1$ is due to the equilibrium assumption on the pilot gases. For Z/D $>5$, a slight overestimation of the temperature is however noticed in the rich flame zone. This behaviour corroborates the observations made on the radial profiles (see Fig. 4). For the inhomogeneous case, the transition between the stratified premixed combustion mode and the nonpremixed mode is well reproduced. However, further downstream, the temperature is slightly overpredicted on a large part of the mixture fraction space.

\section{Conclusions}

In the present paper two premixed based tabulated chemistry combustion models have been used for the simulation of the Sydney modified piloted jet burner. These two models, traditionally used to simulate fully premixed or weakly stratified turbulent flames, have been tested in mixed combustion modes. The prediction of the flame is reasonably well reproduced mainly for the first flame diameters. Further downstream the radial profiles are mispredicted by a too diffusive behaviour of the mixing. When focusing on scatter plots or conditional mean comparisons the flame structure is reasonably well predicted by the two combustion models. Though based on the tabulation of perfectly premixed flame archetype, the combustion models retrieve well the multi-mode combustion regimes in the inhomogeneous flame configuration. The comparison between F-TACLES and TF-LES/FPI models does not show major difference concerning the flame stabilization. However, the simulations using F-TACLES formalism shows a slightly better agreement for the homogeneous case. Further analysis remains to be done on the mixing prediction and on the correct setting of the inflow fluctuation levels. Additional comparisons of $\mathrm{CO}$ emissions would be of interest to assess the capabilities of the two models to predict pollutants in such complex flame configuration.

\section{Acknowledgements}

This project has received funding from the European Unions Horizon 2020 research and innovation programme under the Marie Sklodowska-Curie grant agreement No 643134. This work was granted access to the HPC resources from IDRIS, TGCC and CINES under the allocation 2016-020164 made by GENCI (Grand Equipement National de Calcul Intensif). Prof. Assaad Masri from Sydney University and Dr. 
Robert Barlow from Sandia National Laboratory are warmly acknowledged for sharing the experimental database. Prof. M.E. Muller and B.A Perry from Princeton University are acknowledged for sharing the inflow boundary conditions database.

\section{References}

${ }^{1}$ Fiorina, B., Veynante, D., and Candel, S., "Modeling Combustion Chemistry in Large Eddy Simulation of Turbulent Flames," Flow Turbul. Combust., Vol. 94, 2015, pp. 3-42.

${ }^{2}$ Colin, O., Ducros, F., Veynante, D., and Poinsot, T., "A thickened flame model for large eddy simulations of turbulent premixed combustion," Phys. Fluids, Vol. 12, No. 7, 2000, pp. 1843-1863.

${ }^{3}$ Kuenne, G., Ketelheun, A., and Janicka, J., "LES modeling of premixed combustion using a thickened flame approach coupled with FGM tabulated chemistry," Combustion and Flame, Vol. 158, No. 9, 2011, pp. 1750 - 1767.

${ }^{4}$ Ketelheun, A., Kuenne, G., and Janicka, J., "Heat Transfer Modeling in the Context of Large Eddy Simulation of Premixed Combustion with Tabulated Chemistry." Flow, Turbulence and Combustion, Vol. 91, No. 867-893, 2013.

${ }^{5}$ Fiorina, B., Vicquelin, R., Auzillon, P., Darabiha, N., Gicquel, O., and Veynante, D., "A filtered tabulated chemistry model for LES of premixed combustion," Combustion and Flame, Vol. 157, No. 3, 2010, pp. 465-475.

${ }^{6}$ Auzillon, P., Gicquel, O., Darabiha, N., Veynante, D., and Fiorina, B., "A Filtered Tabulated Chemistry model for LES of stratified flames," Combust. Flame, Vol. 159, No. 8, 8 2012, pp. 2704-2717.

${ }^{7}$ Mercier, R., Auzillon, P., Moureau, V., Darabiha, N., Gicquel, O., Veynante, D., and Fiorina, B., "LES modeling of the impact of heat losses and differential diffusion on turbulent stratified flame: application to the TU Darmstadt stratified flame," Flow Turbul. Combust., Vol. 93, No. 2, 2014, pp. 349-381.

${ }^{8}$ Fiorina, B., Mercier, R., Kuenne, G., Ketelheun, A., Avdić, A., Janicka, J., Geyer, D., Dreizler, A., Alenius, E., Duwig, C., et al., Combustion and Flame, Vol. 162, No. 11, 2015, pp. 4264-4282.

${ }^{9}$ Mercier, R., Schmitt, T., Veynante, D., and Fiorina, B., "The influence of combustion SGS submodels on the resolved flame propagation. Application to the LES of the Cambridge stratified flames," Proceedings of the Combustion Institute, Vol. 35, No. 2, 2015, pp. 1259-1267.

${ }^{10}$ Masri, A., "Partial premixing and stratification in turbulent flames," Proceedings of the Combustion Institute, Vol. 35, No. 2, 2015, pp. 1115-1136.

${ }^{11}$ Lefebvre, A. H., Gas turbine combustion, CRC press, 1998.

${ }^{12}$ Gicquel, L. Y., Staffelbach, G., and Poinsot, T., "Large eddy simulations of gaseous flames in gas turbine combustion chambers," Progress in Energy and Combustion Science, Vol. 38, No. 6, 2012, pp. 782-817.

${ }^{13}$ Franzelli, B., Fiorina, B., and Darabiha, N., Proceedings of the combustion institute, Vol. 34, No. 1, 2013, pp. $1659-1666$.

${ }^{14}$ Barlow, R., Meares, S., Magnotti, G., Cutcher, H., and Masri, A., Combustion and Flame, Vol. 162, No. 10, 2015, pp. $3516-3540$.

${ }^{15}$ Perry, B. A., Mueller, M. E., and Masri, A. R., "A Two Mixture Fraction Flamelet Model for Large Eddy Simulation of Turbulent Jet Flames with Inhomogeneous Inlets," APS Division of Fluid Dynamics Meeting Abstracts, 2015.

${ }^{16}$ Kleinheinz, K., Kubis, T., Trisjono, P., Bode, M., and Pitsch, H., "Computational study of flame characteristics of a turbulent piloted jet burner with inhomogeneous inlets," Proceedings of the Combustion Institute, 2016.

${ }^{17}$ Galindo, S., Salehi, F., Cleary, M., and Masri, A., "MMC-LES simulations of turbulent piloted flames with varying levels of inlet inhomogeneity," Proceedings of the Combustion Institute, 2016.

${ }^{18}$ G.P. Smith, D.M. Golden, M. F. e. a., "GRI3.0 website," http://www.me.berkeleley.edu/gri-mech/.

${ }^{19}$ Pons, L., Darabiha, N., Candel, S., Ribert, G., and Yang, V., "Mass transfer and combustion in transcritical non-premixed counterflows," Combustion Theory and Modelling, Vol. 13, No. 1, 2009, pp. 57-81.

${ }^{20}$ Gicquel, O., Darabiha, N., and Thévenin, D., Proceedings of the Combustion Institute, Vol. 28, No. 2, 2000, pp. 1901-1908.

${ }^{21}$ Fiorina, B., Baron, R., Gicquel, O., Thevenin, D., Carpentier, S., Darabiha, N., et al., "Modelling non-adiabatic partially premixed flames using flame-prolongation of ILDM," Combustion Theory and Modelling, Vol. 7, No. 3, 2003, pp. 449-470.

${ }^{22}$ Peters, N., Turbulent combustion, Cambridge university press, 2000.

${ }^{23}$ Poinsot, T. and Veynante, D., Theoretical and numerical combustion, RT Edwards, Inc., 2012.

${ }^{24}$ Charlette, F., Meneveau, C., and Veynante, D., "A power-law flame wrinkling model for LES of premixed turbulent combustion Part II: dynamic formulation," Combustion and Flame, Vol. 131, No. 1, 2002, pp. 181-197.

${ }^{25}$ Wang, G., Boileau, M., and Veynante, D., Combustion and Flame, Vol. 158, No. 11, 2011, pp. 2199-2213.

${ }^{26} \mathrm{~B}$.Franzelli, Impact of the chemical description on direct numerical simulation and large eddy simulation of turbulent combustion in industrial aero-engines, Ph.D. thesis, Universit de Toulouse, 2011.

${ }^{27}$ Auzillon, P., Gicquel, O., Darabiha, N., Veynante, D., and Fiorina, B., Combustion and Flame, Vol. 159, No. 8, 2012, pp. 2704-2717.

${ }^{28}$ Meares, S. and Masri, A. R., Combustion and Flame, Vol. 161, No. 2, 2014, pp. $484-495$.

${ }^{29}$ Moureau, V., Domingo, P., and Vervisch, L., Comptes Rendus Mécanique, Vol. 339, No. 2-3, 2011, pp. $141-148$.

${ }^{30}$ Germano, M., Piomelli, U., Moin, P., and Cabot, W. H., "A dynamic subgrid-scale eddy viscosity model," Physics of Fluids A: Fluid Dynamics (1989-1993), Vol. 3, No. 7, 1991, pp. 1760-1765.

31 "TNF2016 Workshop," http://www.sandia.gov/TNF/13thWorkshop/index.php.

${ }^{32}$ Auzillon, P., Fiorina, B., Vicquelin, R., Darabiha, N., Gicquel, O., and Veynante, D., "Modeling chemical flame structure and combustion dynamics in LES," Proceedings of the Combustion Institute, Vol. 33, No. 1, 2011, pp. 1331-1338. 

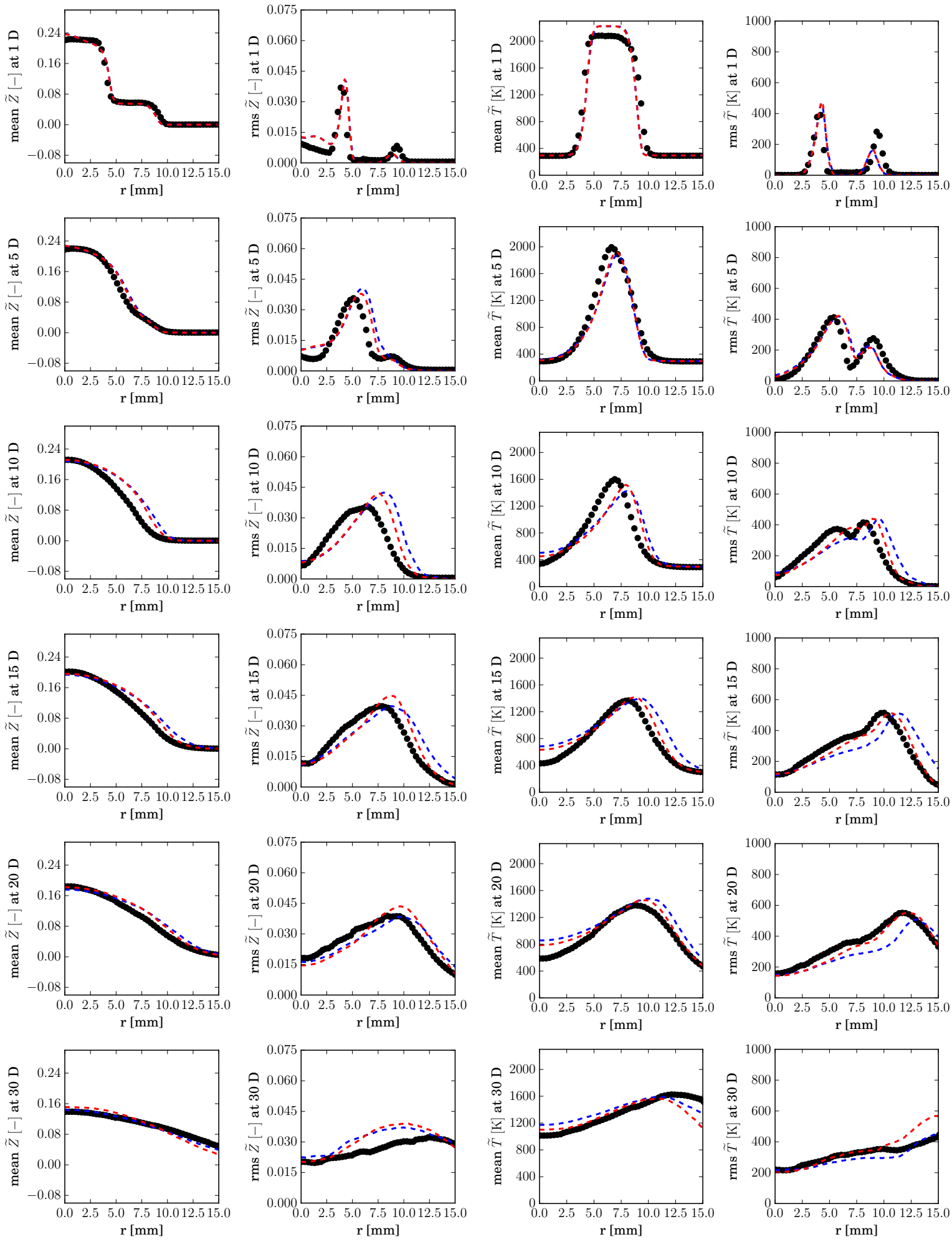

Figure 4. Radial profile of mean and rms of temperature (right column) and mixture fraction (left column) for different axial positions respect to the burner exit plane. Comparison of the experimental values respect to the simulation results obtained for the configuration FJ200 - 5GP - Lr300 - 59. Legend: - - F-TACLES model. - - TFLES-FPI model. • • • Sandia measurements 

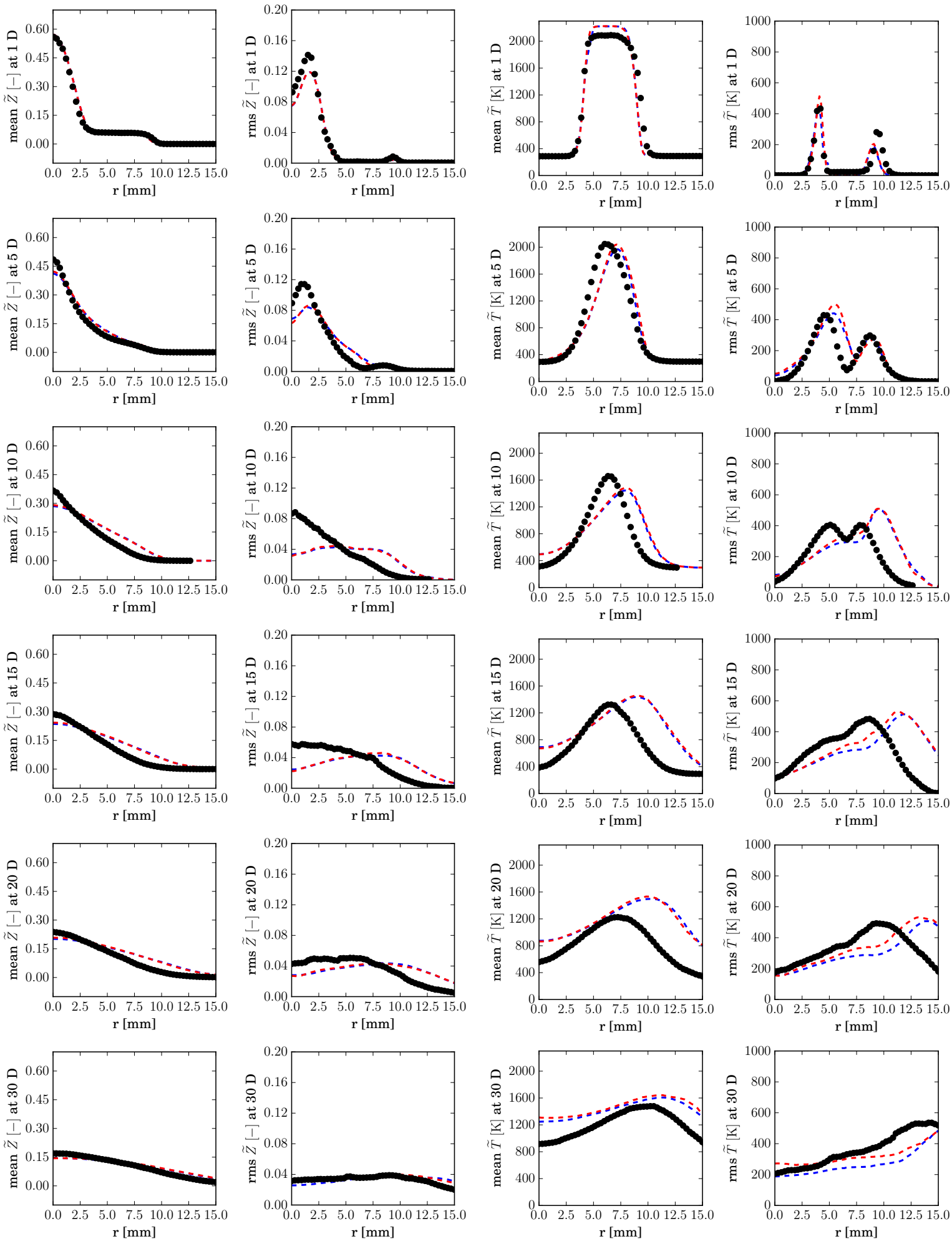

Figure 5. Radial profile of mean and rms of temperature (right column) and mixture fraction (left column) for different axial positions respect to the burner exit plane. Comparison of the experimental values respect to the simulation results for the configuration FJ200 - 5GP - Lr75 - 80. Legend: - - F-TACLES model. - TFLES-FPI model. •• Sandia measurements 

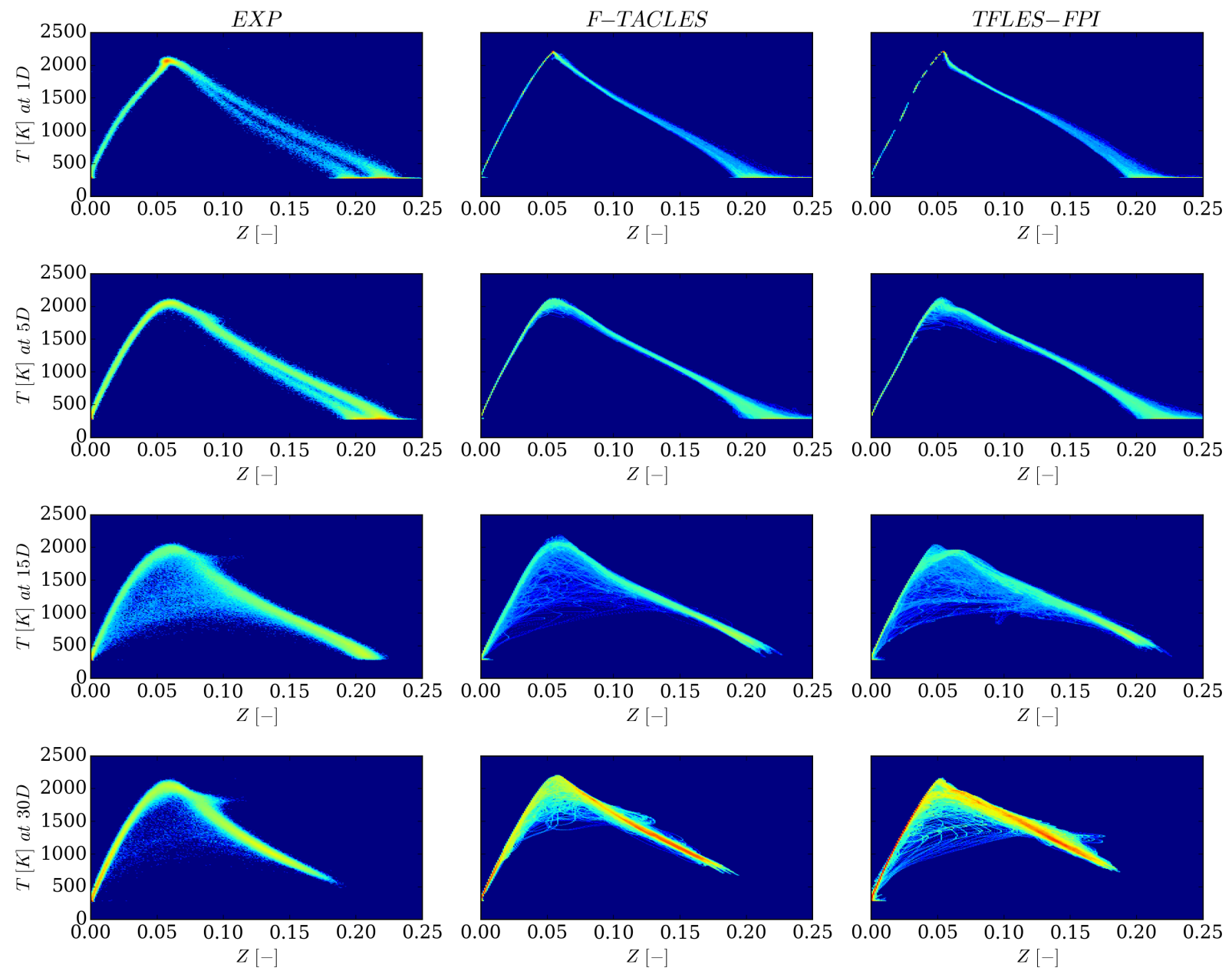

Figure 6. Instantaneous trajectories covered by the flame in the space temperature versus mixture fraction for various axial positions for the case FJ200-5GP-Lr300-59. Experimental data are compared with the simulation results obtained with the two combustion models. The data are colored by the local normalized density of points. The normalization value is the total number of points considered. The red color corresponds to a normalized density grater than 0.3 while the blue color correspond to a normalized density lower than $10 \mathrm{E}-6$. 

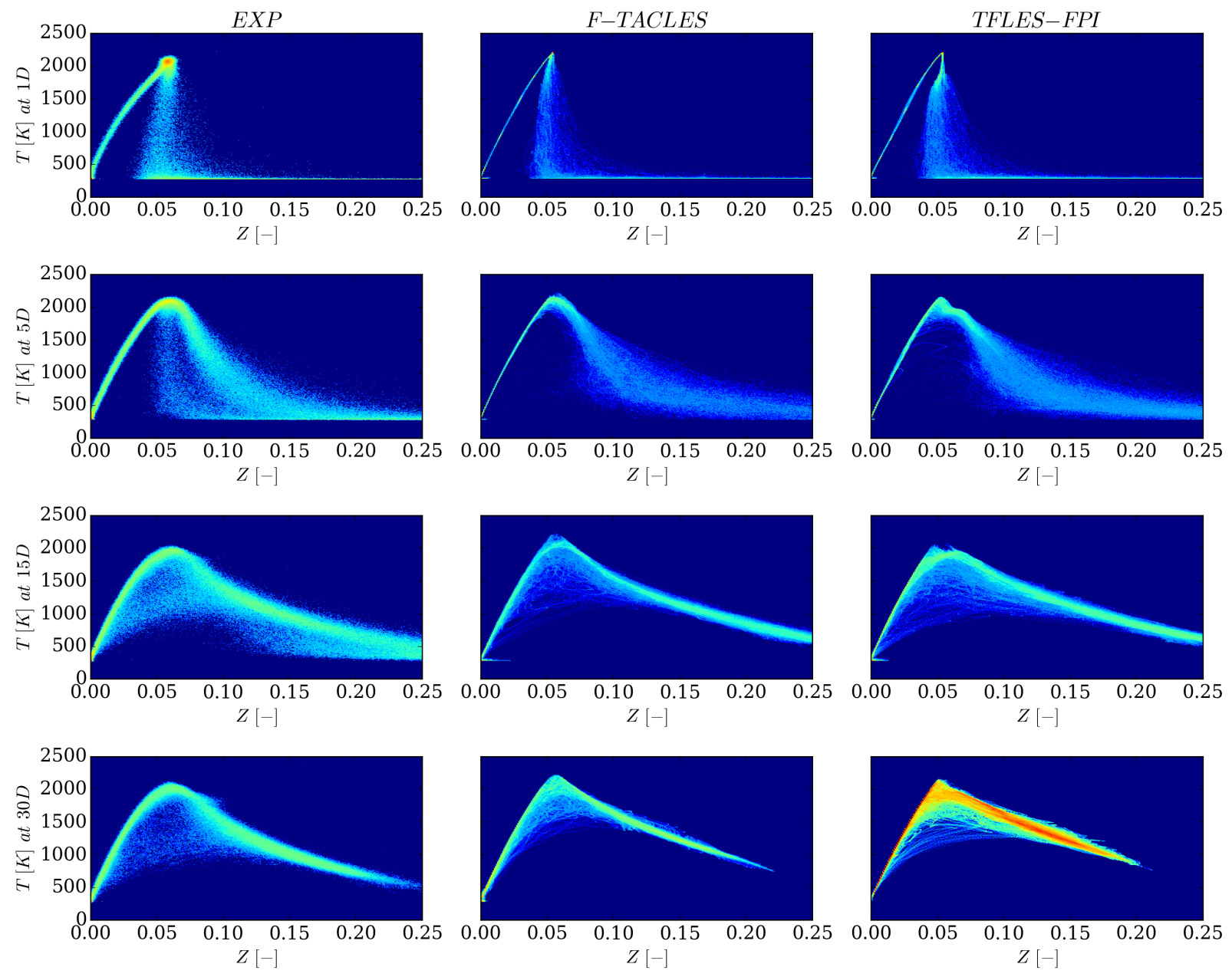

Figure 7. Instantaneous trajectories covered by the flame in the space temperature versus mixture fraction for various axial positions for the case FJ200-5GP-Lr75-80. Experimental data are compared with the simulation results obtained with the two combustion models. The data are colored by the local normalized density of points. The normalization value is the total number of points considered. The red color corresponds to a normalized density grater than 0.3 while the blue color correspond to a normalized density lower than $10 \mathrm{E}-6$. 

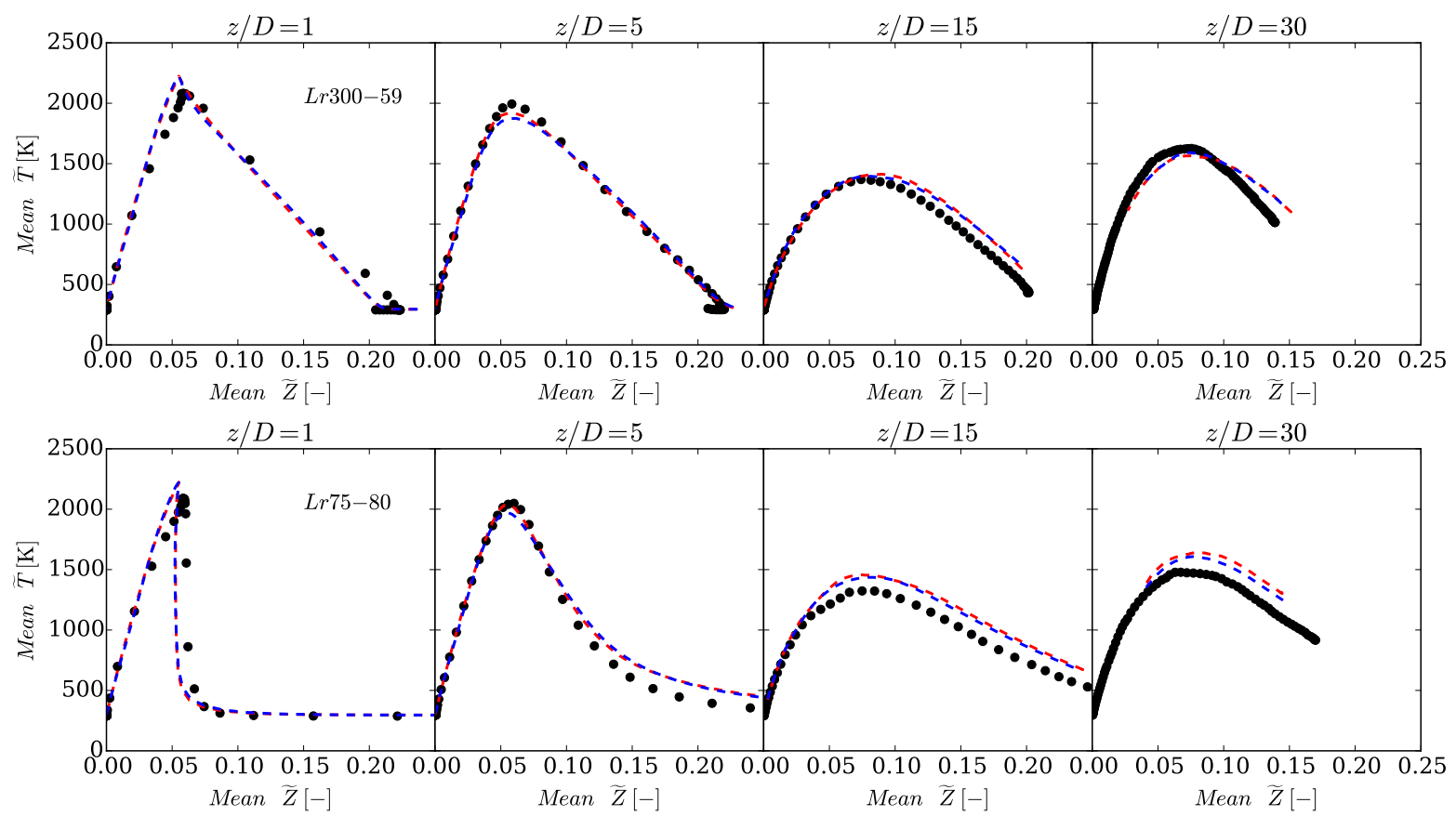

Figure 8. Conditional temperature mean respect to mixture fraction mean for various axial positions downstream the burner exit plane. The upper figure refers to the homogeneous case. The lower figure refers to the inhomogeneous case. Legend: - - F-TACLES model. - - TFLES-FPI model. • • Sandia measurements 